\section{Keratolysis exfoliativa-like eruption induced by ranolazine}

\author{
Elena Pierobon, ${ }^{1}$ Lerica Germi, ${ }^{2}$ \\ Andrea Sechi, ${ }^{2}$ Giampaolo Trevisan, ${ }^{2}$ \\ Elena Pezzolo, ${ }^{2}$ Claudio Feliciani, ${ }^{1}$ \\ Luigi Naldi ${ }^{2}$
}

${ }^{1}$ Section of Dermatology, Department of Medicine and Surgery, University of

Parma; ${ }^{2}$ Dermatology Unit, San Bortolo

Hospital, Vicenza, Italy

\begin{abstract}
Dermatoses affecting palms may represent a dermatologic challenge from both the diagnostic, and therapeutic point of view. Patients with supposedly occupational dermatitis can spend months or even years in a frustrating attempt to avoid contact with possible irritants or allergens. To underline the importance of a thorough unbiased analysis of the patient's history and clinical features, we present the iconic case of a bricklayer affected by a chronic, disabling desquamation of palms which in the end was classified as keratolysis exfoliativa (KE) attributed to ranolazine-intake, an antianginal drug. To the best of our knowledge, this specific adverse effect of druginduced KE of palms has never been reported before in association with ranolazine.
\end{abstract}

\section{Case Report}

We report the case of a 58 old man that was referred to our Dermatological Unit presenting a 2-years history of painful desquamation of both palms of the hands. He had been working for over 20-years as a bricklayer and the skin condition was particularly disabling interfering with ordinary life and specific job tasks.

At the dermatological examination both hands appeared affected only on the palms, showing ill-defined collarettes of dry, peeled areas with tendency to painful fissures, resembling a keratolysis exfoliativa (KE) (Figure 1).

He had no evidence of other dermatological signs or symptoms and had no previous history or hereditary predisposition for cutaneous conditions, including psoriasis and atopic dermatitis. His medical history highlighted a cardiac ischemic disease for which he had for many years a chronic therapy with antihypertensive and cholesterol-lowering medicines to which had been added 2 years before a new therapy with ranolazine to treat chronic stable angina pectoris.

Suspecting a chronic irritant contact dermatitis (ICD), we prescribed topical steroids to relieve the inflammation, suggested the use of protective gloves, barrier creams and moisturisers, providing also the appropriate information to prevent exposure to occupational irritants. The patient carefully applied our indications but at the follow up visit his dermatitis seemed to have no improvement at all. We questioned the diagnosis of ICD and focused our attention to a possible delayed-type hypersensitivity reaction. At patch testing our patient was completely negative for the most frequent haptens for general population and building works (potassium dichromate, tiuram mix and nickel sulphate).

Far from ready to give up, we underlined the temporal link from the first ranolazine intake and the onset of hand desquamation, both 2 years before. We suggest changing the cardiological therapy and after one month of ranolazine suspension, the patient's skin recovered fully (Figure 2).

\section{Discussion}

Hand dermatoses are an extremely frequent cause of dermatological consultation and may be a consequence of lots of possible agents, exogenous or endogenous, supported by irritative, allergic or infective agents. Frequently the clinical distribution and morphology of lesions are not enough specific to make the diagnosis because of a consistent risk of clinical mimicking of different aetiology. Collecting an accurate physiological anamnesis and medical history can be extremely important to investigate the role of constitutional predisposition and exogenous factors. The skin is the primary direct interface with external agents and patients whose lesions affect the hands alone are likely to suffer from an occupational contact dermatitis ${ }^{1}$. Considering daily contact of our patient with wet cement, clinical features, pain and burning sensation, our case was highly suggestive for an irritant chronic eczema (ICD). ICD is caused by chemical or physical skin damage and represents approximately $80 \%$ of all cases of occupational skin disorder. ${ }^{1}$

At the failure of the first hypothesis, the second proposed diagnosis was equally possible: a minority of occupational dermatitis are allergic contact dermatitis (ACD) caused by a delayed-type hypersensitivity reaction. ${ }^{1}$ Building workers can be commonly exposed to allergens which can be detected by a properly performed patch test-
Correspondence: Elena Pierobon, Section of Dermatology, Department of Medicine and Surgery, University of Parma, Via Gramsci 14, 43126 Parma, Italy.

Tel.: +39.0521.702711 - Fax: +39.0521.702959.

E-mail: elena.pierobon@studenti.unipr.it

Key words: Keratolysis exfoliativa, Ranolazine, Cutaneous drug reaction, Hand dermatititis.

Contributions: EPi wrote most of the original draft of the paper. LG participated in drafting and designing the study. AS, EPe and GT had access to all the raw data of the study and participated in generating and gathering them. $\mathrm{LN}$ and CF revised the work critically for important intellectual content and gave their contribute in the process of analysis and interpretation of data for the work. All authors participated in writing the paper and have approved the final version.

Conflict of interest: The authors declare no potential conflict of interest.

Funding: None.

Availability of data and material: Data and materials are available by the authors.

Please cite this article as: Pierobon E, Germi L, Sechi A, et al. Keratolysis exfoliativa-like eruption induced by ranolazine. Dermatol Rep 2022;14:9264.

Received for publication: 13 May 2021. Accepted for publication: 12 July 2021.

This work is licensed under a Creative Commons Attribution-NonCommercial 4.0 International License (CC BY-NC 4.0).

Copyright: the Author(s), 2022

Licensee PAGEPress, Italy

Dermatology Reports 2022; 14:9264

doi:10.4081/dr.2022.9264

ing. Not at least, lesions that are clinically suggestive for an infectious aetiology, mycotic or bacterial, can be easily ruled out thanks to microscopic and culture examination. ${ }^{2}$ As these 3 most probable hypotheses came down, it is correct to focus the attention on the patient overall health, and consider of an endogenous mechanism, such as atopic dermatitis. Although less frequent, dermatologists should also consider the possibility of a drug reaction. In our case, ranolazine administration was started one month before the onset of the disease, and we suspected an acral drug-induced manifestation. The antianginal drug was interrupted obtaining a rapid restitutio ad inte- 


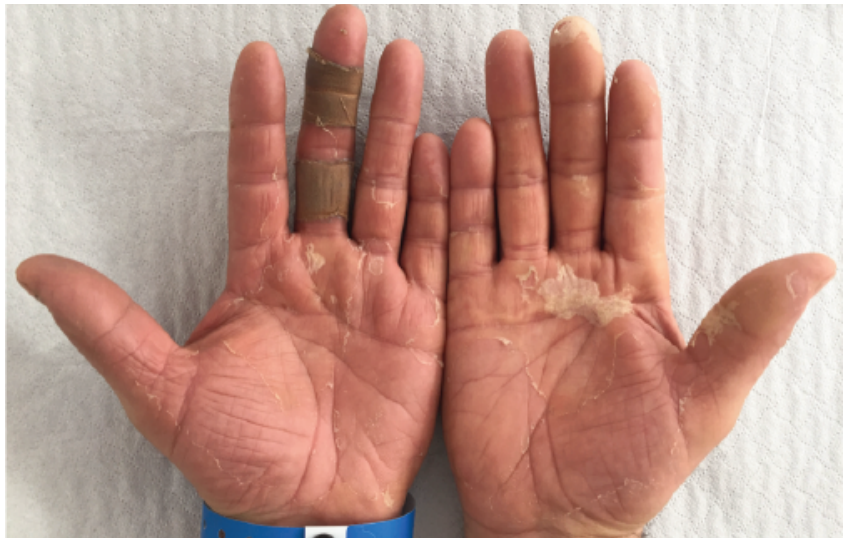

Figure 1. Exfoliative dermatitis of the palms characterized by expanding collarettes of focal peeling of the stratum corneum.

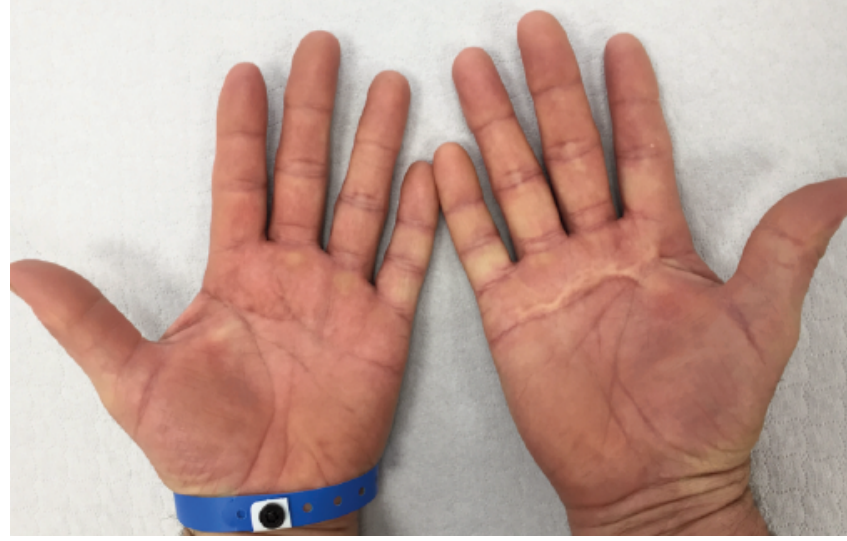

Figure 2. Complete restitutio ad integrum after suspension of cardiologic therapy with ranolazine. grum which allows us to confirm the diagnosis of a KE-like reaction to ranolazine. Palmoplantar exfoliation can be a consequence of genetic defects, as happens in acral peeling skin syndrome, or can be triggered by infections, as tinea pedis and scarlet fever. ${ }^{3}$ Less frequently it can be a localized manifestation of a cutaneous drug reaction, ${ }^{3}$ which may comprehend a variety of conditions that can affect acral vasomotility, hand pigmentation, skin and nails structure. ${ }^{2}$ Many chemotherapeutic agents and molecular targeted therapies can cause hand-foot syndrome, or erythrodysesthesia, which is an erythematous- desquamative and painful condition of palms and soles. ${ }^{4}$ Cardiologic therapy can rarely cause palmo-plantar desquamation and literature provides evidence of few cases triggered by calcium channel blockers, especially diltiazem, amiodarone, enalapril and phenindione. ${ }^{5}$ In our case we formulated the clinical diagnosis of $\mathrm{KE}$, also known as dyshidrosis lamellosa sicca. ${ }^{6}$ The condition is characterized by small, annular collarettes of white desquamation involving only the palms (and, less commonly, the soles) of hands and feet. ${ }^{6}$ As in our case, mechanical friction and water contact may exacerbate the cleavage and partially degradation of corneodesmosomes within the stratum corneum, and the absence of an inflammatory infiltrate is characteristic. ${ }^{6}$ The discrete appearance of desquamation, limited to palms, the adult onset and the absence of inflammation signs allowed us to exclude both acral peeling skin and ery- throdysesthesia. KE is a common but frequently under-recognized peeling entity with uncertain origin, and both sporadic and familial cases have been described. ${ }^{6}$ To our knowledge, literature offers evidence of only one other case of drug-induced KE, triggered by chloroquine intake. ${ }^{3}$ We herein propose the first report of a KE-like eruption to ranolazine, an antianginal molecule which selectively inhibits the late sodium current. ${ }^{7}$ Except for the possibility of an immediate hypersensitivity reaction and urticaria onset, there are few evidence about ranolazine cutaneous side effects. Common side effects include dizziness, nausea and constipation while peripheral oedema, headache, asthenia, palpitations, dyspepsia, weakness and postural hypotension are less frequently reported. ${ }^{8}$

\section{Conclusions}

Mild cutaneous drug reactions are insidious and challenging diagnoses because of the high risk of mimicking other dermatological patterns that can be more frequent in specific contexts. In our opinion, dermatologists should always keep the focus on the possibility of a drug reaction also when clinical manifestations are limited to a part of the body, as happens in fixed erythema. We suggest to collect a detailed and complete pharmacological history during every medical examination as a good approach to patient care in everyday clinical practice.

\section{References}

1. Sasseville D. Occupational contact dermatitis. Allergy Asthma Clin Immunol 2008;4:59-65.

2. Caccavale S, Ruocco E. Acral manifestations of systemic diseases: druginduced and infectious diseases. Clin Dermatol 2017;35:55-63.

3. Nair PA, Patel T. Palmoplantar exfoliation due to chloroquine. Indian J Pharmacol 2017;49:205-7.

4. Nikolaou V, Syrigos K, Saif MW. Incidence and implications of chemotherapy related hand-foot syndrome. Expert Opin Drug Saf 2016;15:1625-33.

5. Toescu SM, Kennon S, Stevens H. Diltiazem-induced palmar desquamation and oral erosions. BMJ Case Rep 2013:bcr2013201536.

6. Chang YY, van der Velden J, van der Wier $G$, et al. Keratolysis exfoliativa (dyshidrosis lamellosa sicca): a distinct peeling entity. Br J Dermatol 2012;167: 1076-84.

7. Rayner-Hartley E, Sedlak T. Ranolazine: a contemporary review. J Am Heart Assoc 2016;5:e003196.

8. Salazar CA, Basilio Flores JE, Veramendi Espinoza LE et al. Ranolazine for stable angina pectoris. Cochrane Database Syst Rev 2017;2:CD011747. 